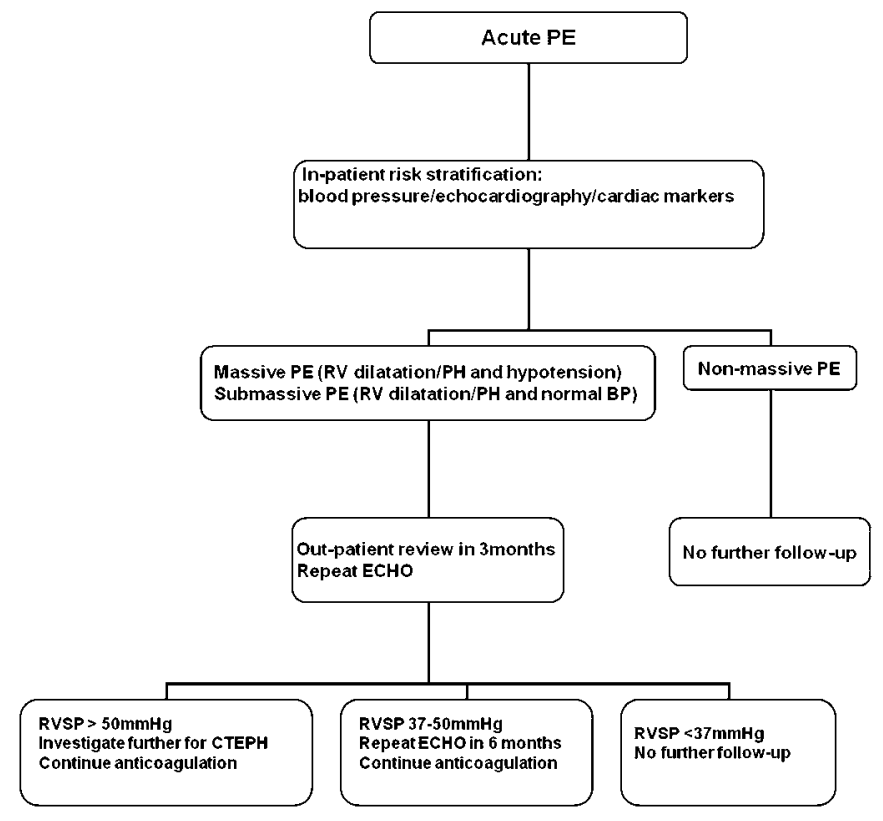

Abstract P7 Figure 1

\section{P8 USE OF D-DIMER: CRP RATIO COMPARED TO D-DIMER ALONE TO PREDICT PE ON VO SCANNING}

doi:10.1136/thoraxjnl-2011-201054c.8

R Berwick, S Navalkissoor, J R Hurst. UCL Medical School, London, UK

Introduction Pulmonary embolism (PE) is a common presentation in the emergency department and in-patient setting. Measurement of D-dimer in conjunction with clinical risk assessment is used to exclude patients at low risk of PE. Some of the conditions that mimic PE, including infection and inflammation, are also associated with elevated D-dimer concentrations such that the test lacks specificity. Most infectious and inflammatory conditions result in an elevated acute-phase serum response which can be quantified using C-Reactive Protein (CRP) assay. We hypothesised, therefore, that patients with isolated $\mathrm{PE}$ would have a higher $\mathrm{D}$-dimer: CRP ratio than patients with infectious or inflammatory mimics of PE and therefore that this ratio would be more discriminatory.

Methods We analysed data from all patients who underwent V/O scanning to confirm or exclude PE at Royal Free Hampstead NHS Trust, London, UK, during 2010. The CRP and D-dimer results closest, but preceding the $\mathrm{V} / \mathrm{Q}$ scan were analysed using receiver operator characteristic (ROC) curves to test the hypothesis that the D-dimer: CRP ratio (expressed as $\mathrm{ng} / \mathrm{ml}: \mathrm{mg} / \mathrm{l}$ ) was a better predictor or PE than D-dimer alone.

Results 179 patients (mean (SD) age 52.8 (19.7) years) had a V/O scan for suspected PE during the study period. Of these, 85 had a Ddimer assay, a median (IOR) of $1(0-1)$ days prior to the imaging. The median D-dimer concentration was 272 (178-675) ng/ml. 137 patients had CRP assay $(12(3-56) \mathrm{mg} / \mathrm{l})$, measured $1(0-1)$ days prior to imaging. It was possible to calculate a D-dimer: CRP ratio in 78 patients (44\% of the total), of whom 19 (24\%) had a V/O scan reported as high risk for PE. D-dimer, and the D-dimer: CRP ratio, but not CRP were significantly higher between patients who did and did not have high-risk V/O scans (Mann-Whitney $U$ test analyses: 764 vs $245 \mathrm{ng} / \mathrm{ml}, \mathrm{p}=0.001 ; 107$ vs 31 units, $\mathrm{p}=0.020$ and 20 vs $10 \mathrm{mg} / \mathrm{l}, \mathrm{p}=0.134$ respectively). Biomarker data were $\log _{10}$ transformed to permit ROC analysis. Area-under-curve (AUC) values using ROC for $\mathrm{D}$-dimer alone, and $\mathrm{D}$-dimer: $\mathrm{CRP}$ ratio were 0.74 and 0.68 respectively, both less than the standard criteria for utility of 0.8 .
Conclusions D-dimer: CRP ratio is not superior to D-dimer alone in predicting $P E$ in patients with a clinical suspicion of this diagnosis sufficient to require $\mathrm{V} / \mathrm{Q}$ scanning.

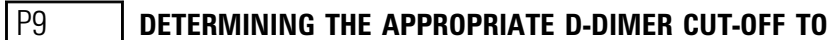 EXCLUDE PULMONARY EMBOLI IN AN AMBULATORY CARE SETTING USING DIFFERENT THRESHOLDS BASED ON PRE- TEST PROBABILITY}

doi:10.1136/thoraxjnl-2011-201054c.9

R M Ladwa, E Bailie, Y Vali, R H Green, J A Bennett, C M Free. University Hospitals of Leicester, Leicester, UK

Introduction Currently the same threshold value is used to identify a positive D-dimer result for all patients presenting to our ambulatory clinic with suspected pulmonary emboli (PE). It has been suggested that adjusting the threshold value according to the pre-test probability would exclude PE in more patients than using the same cutoff point regardless of clinical probability.

Methods Data from 362 consecutive patients presenting to the ambulatory PE clinic was collected. A pre-test probability of PE was recorded for all patients and those with a high pre-test probability had radiological investigations. Patients with a low or intermediate pre-test probability had a latex agglutination $\mathrm{D}$-dimer test. If this result was $=0.5 \mu \mathrm{g} / \mathrm{ml}$ they had further investigations, otherwise they were discharged. The diagnosis of PE was made if a VQ scan showed ventilation/perfusion mismatch or CTPA report demonstrated PE. Receiver operating characteristic curve analysis was performed separately for patients with low and intermediate probability and the optimum cut-off value to exclude PE determined. Sensitivity, specificity, negative predictive value and positive predictive value for different cut-off points were determined.

Results 362 patients were included in the analysis, 207 (57\%) had low, $129(36 \%)$ intermediate and $26(7 \%)$ high pre-test probability. Prevalence of PE was $2 \%$ in the low probability group, $14 \%$ in the intermediate probability group and $42 \%$ in the high probability group. No patients with a D-dimer of $<0.5 \mu \mathrm{g} / \mathrm{ml}$ who were discharged without further tests have re-presented with similar symptoms. In the low pre-test probability group, a cut-off point of 1.07 improved the specificity from $64 \%$ to $89 \%$ while maintaining a sensitivity of $100 \%$ and negative predictive value of $100 \%$. Analysis in patients in the intermediate risk group suggested that a cut-off of $0.5 \mu \mathrm{g} / \mathrm{ml}$ was appropriate. By adjusting the D-dimer threshold to $>1.0 \mu \mathrm{g} / \mathrm{ml}$ in the low probability group, a further 53 patients could have been discharged home without need for radiological investigation.

Conclusion The diagnostic accuracy of D-dimer testing may be improved in patients with a low pre-test probability by adjusting the cut-off threshold.

\section{P10 RISKS OF LOW MOLECULAR WEIGHT HEPARIN IN SUSPECTED PULMONARY EMBOLISM}

doi:10.1136/thoraxjnl-2011-201054c.10

L Watkins, S Rafeeq, N McMullan, P Stockton, S Twite, S Agarwal. St Helens and Knowsley Teaching Hospitals NHS Trust, Prescot, UK

Background National Patients Safety Agency (NPSA) issued a statement in July 2010 highlighting the risks associated with the prescription of low molecular weight Heparins (LMWHs). Evidence of harm has been reported due to dosing errors caused by failure to weigh patients and calculate creatinine clearance.

Aim We hypothesised that harm associated with prescription of LMWHs is underreported on the national reporting and learning system (NRLS). We performed a retrospective study to evaluate the frequency of harm associated with LMWHs in patients admitted with a suspicion of pulmonary embolism (PE). 